Article

\title{
On the Complex Interaction between Collective Learning and Social Dynamics
}

\author{
Diletta Burini ${ }^{1, *,+(D)}$ and Silvana De Lillo ${ }^{1,2,+}$ \\ 1 Department of Mathematics and Computer Science, University of Perugia, 06123 Perugia, Italy \\ 2 Istituto Nazionale di Fisica Nucleare, 06123 Sezione di Perugia, Italy \\ * Correspondence: dilettaburini@alice.it \\ + These authors contributed equally to this work.
}

Received: 28 June 2019; Accepted: 22 July 2019; Published: 1 August 2019 updates

\begin{abstract}
This paper is motivated by the perspective ideas proposed in our previous studies, where some challenging problems, for instance qualitative analysis of the solution to nonlinear problems and micro-macro asymptotic analysis, where posed. Our work focuses on the study of the interactions between learning dynamics and other types of dynamics which can be modeled by kinetic theory methods. The contents are presented in three parts. First, a general description of different theories of learning dynamics within the framework of cognitive sciences is critically analyzed with the aim of capturing the main features of the system towards modeling. Subsequently, the class of systems which are the object of the modeling approach is defined by showing how the previous structure can be developed, thanks to new conceptual ideas, including the concept of symmetric and asymmetric learning, towards modeling. Finally, some applications are selected to show how the approach can be methodologically applied.
\end{abstract}

Keywords: learning dynamics; kinetic theory; complex systems

\section{Plan of the Paper}

A mathematical approach to the collective learning dynamics has been developed in [1-3] to model individual and collective learning by interactions involving individuals and groups of individuals. The method combines the mathematical tools of the kinetic theory of active particles [4] and the theoretical tools of evolutionary game theory $[5,6]$. In more detail, the sequential steps of the approach are as follows:

1. The level of learning of a certain knowledge is modeled, at the microscopic level, by a scalar variable $u \in[0,1]$, where $u=0$ represents the lowest level of achieved knowledge and $u=1$ the highest admissible level.

2. The whole system can be subdivided into functional subsystems, in which each one of them plays a different role in the learning process.

3. The overall state of the system is delivered, i.e., a probability distribution function over the microscopic state defines the collective state of the system, while macroscopic states are provided by weighted moments of this probability distribution.

4. The dynamics develops through encounters which can be either individual-based, or between individuals and the whole system.

5. The output of the interactions is modeled by developments of the theoretical tools of the evolutionary game theory, in which players are probability distributions or their averaged quantities. 
6. The probability distribution, mentioned in Item 3, is the dependent variable of a differential system. Its dynamics is obtained by a balance of "living" particles in the elementary volume of the microscopic states.

The interested reader is referred to [1] for additional information on the modeling approach, to the survey [7] and to the book [4] for the related mathematical tools, while additional details will be given in the next sections.

The contents of our paper rely on the observation that the perspective ideas proposed in [1] leave open various problems that are definitely worth of future investigations. In more detail, this present paper focuses on the study of the interactions between learning dynamics and other types of dynamics that can be accounted for by kinetic theory tools. In fact, some recent papers have put in evidence how models which involve interactions of different dynamics can generate even outputs that could not be foreseen, but which are confirmed in reality.

A possible example is given in [8], where it is shown that an unfair wealth policy can lead to a radicalization of the opposition to the governments which has produced such policy. The opposition also ends up involving the social classes that apparently take advantage of the policy, however unfair it is. Further applications are suggested in [9], focusing on the interactions between learning and behavioral economy. An additional example is the study of the complex interaction between learning in crowd dynamics, in which the walking strategy is induced by learning social-emotional behaviors.

These examples, discussed in the last section, motivate the contents of this paper which is devoted to the modeling of the influence of learning dynamics on other types of dynamics. This is the main novelty of our paper, where, in addition, the use of discrete states of learning dynamics and of the variables of subsequent dynamics is developed, also exploiting a new metrics to model the distance between the interacting entities. The contents are proposed through three other sections.

Section 2 provides a general description of the different theories of learning dynamics in the framework of cognitive sciences. Some specific issues are extracted, without claim of completeness, from a vast and heterogeneous literature that is still waiting for a unified interpretation. This section attempts to open a dialogue between mathematics and cognitive sciences looking forward to a unified approach generated by the mathematical sciences.

Section 3 defines the specific systems to be modeled and then shows how the approach proposed in [1] can be developed, thanks to new conceptual approaches, towards the modeling of the class of systems presented in Section 2. The conceptual novelties consist of using the discrete probability distribution to describe the state of the system and the discrete stochastic games to model the interactions, and in dealing with a hierarchy where learning precedes a consequent dynamics.

Section 4 deals with applications, selected among several possible ones, to show how the approach can be specifically referred to modeling. These applications specifically refer to the two aforementioned dynamics, firstly the modeling of wealth policy opposition to governments up to radicalization and then the interaction between learning walking strategy in crowd dynamics. Lastly, we consider a third application i.e., the immune competition in heterogenous multicellular systems, where learning leads to proliferative and destructive events. The presentation of these case studies concludes with a critical analysis that, starting from the theoretical achievements of our paper, looks ahead to research perspectives.

\section{State of the Art}

This section introduces topics related to collective learning that constitute the basis for the modeling approach developed in our paper. The contents are presented in three subsections. The phenomenological description of collective learning is treated in the first subsection. An overview of the existing mathematical approaches known in the literature is delivered in the second subsection. The third subsection provides a critical analysis that defines more precisely the objectives of the mathematical method proposed from Section 3. 


\subsection{Phenomenological Description of Collective Learning}

We start examining the interrelations between individual and social learning, which appear as two different aspects of the same phenomenon of collective learning, to clarify how they are interconnected.

The concept of individual learning has come to be embedded into social and cultural contexts and interactions, due to the growing interest in Vygotsky's theory [10], to retrospective examinations of the role of social interactions in [11], and in the subsequent Bandura's revisiting [12]. Two strands are involved: the "cognitive, acquisition-oriented" conception of individual learning with respect to the "situative, participatory" concept [13]. Learning occurs in individuals' minds and, as we elaborate later, it takes place as a social and participatory process, offering two distinctively different points of view on learning.

The idea of social learning originated with the development of psychology sciences [14] and occurs when the individual learns new behaviors and concepts from others. Because of its lack of rigor, it has been ignored by psychologists over the years, and it has been relegated, at best, to the study of the background context, not exactly on a par with the learning of the individual [15]. Social learning is often combined with other dynamics, for instance participation and pro-environmental behavior, while, in general, small differences are referred to individual and social learning.

After this general presentation, we now provide, referring to [16], some characterizations of social learning that can be useful for the modeling process that we want to develop later. The first aspect to keep into account is the active social mediation of individual learning: a person or a team helps an individual to learn e.g., a teacher and a child forming a joint learning system.

Another important characterization, which deserves to be mentioned, consists of considering the social entity as a learning system. A good example is the case of a collective agency that, as a collective, acquires more knowledge, understanding, or skill e.g., a business organization develops internal procedures that satisfy the efficiency of customer requests.

Finally, the last aspect we wish to point out is learning to be a social learner: an important dimension of learning to learn is how that participate in and capitalize on the social milieu.

Over the years, a frequent confusion has emerged between the concept of social learning itself and its potential outcomes. The lack of clarity on this matter has reduced the possibility to distinguish precisely the two, however different, types of learning [17]. For these reasons, in [18], the authors try to answer the question: what is social learning?, and they identify three key problems with the term as it is currently used in the literature:

- Social learning can possibly occur even without any previously organized interaction, while social networks can contribute to the interaction dynamics [19-21].

- The quality, namely level of depth, of this type of learning can span from superficial levels to deep conceptual levels which can involve groups at different scales [22,23].

In conclusion, social learning is effective if a change in understanding appears in the individuals involved in the dynamics.

Closely connected with the previous analysis is the concept of collective learning which is a complex dynamics that has been variously defined in different ways. It is mainly viewed as a dynamic and cumulative process.

An interesting example is provided by the deep learning algorithms recently tested in the diagnosis of skin cancers [24]. The algorithm combines visual processing with deep learning, a type of artificial intelligence modeled by models of neural networks. Learning is then an evolutionary process related to the dynamics of collective knowledge [25].

Among the characterizations of the collective learning, we want to bring the analysis done in the context of transfer knowledge in technology milieu, where in [26] the author approaches a definition of collective learning and its interpretation within spatial theories. First, collective learning to be interpreted as a social process of cumulative knowledge [27,28]. 
In conclusion, if learning is to be considered collective learning, then it must be cumulative and interactive. Learning is a dynamic process, developed on the basis of an element of continuity over time, on which knowledge rests and accumulates. Furthermore, the new knowledge of a first inventor is transferred through other agents on the basis of an element of synergy and an interactive process, giving rise to a cumulative process of knowledge creation.

\subsection{A Brief Survey of Mathematical Approaches}

Due to the lack of a socio-psychological theory on social learning, mathematical approaches also differ significantly from one another, waiting for a possible uniformity. In this subsection, we present some approaches, bearing in mind the three sequential steps that occur in the rough simplification of learning dynamics in complex living systems toward modeling. The first step of learning dynamics is the perception of inputs from other individuals. These inputs are perceived within an individual domain within which the said inputs are effectively felt. Thus, interactions take place and trigger a learning process that changes their level of knowledge.

From the point of view of theoretical modeling, very interesting studies have been carried out in the framework of statistical physics methods in recent years. A very broad review concerning this general framework was presented in [29], where the authors focus their attention on different aspects emerging in social dynamics. All such aspects seem to be related to the fundamental issue of how the interactions between social agents allow the emergence of a macroscopic order starting from a disordered initial situation. In [29], the authors focus on some conceptually homogeneous topics, sharing the common aspect according to which individuals are viewed as adaptive rather than rational agents that communicate and interact with each other.

In the context of social dynamics, the agreement is one of its most important aspects. Everyday life presents many situations in which it is necessary for a group to reach shared decisions. One of the pioneer contributions to the modeling agreement/disagreement dynamics, which might be referred to opinion dynamics, has been proposed by a model proposed by Weidlich [30]. Subsequently, a modification of the Ising model has been proposed to model specific features of opinion dynamics [31,32], where the spin-spin coupling is deemed to model pairwise interaction, while a background field represented the cultural majority or propaganda. Subsequently, a branch of opinion dynamic is based on the so-called "voter model" [33], which was first considered in [34] as a model for species competition. Kinetic models of continuous opinion formation involving both the exchange of opinions between individual agents and dissemination of information are proposed in [35-37].

In the framework of stochastic models, the Axelrod model played a prominent role [38], by enlightening the two mechanisms, which, according to his theory, are fundamental in understanding of the dynamics of cultural assimilation, namely social influence and homophily.

Collective learning, on the other hand, has a very strong influence also in the modeling of collective motion. This latter appears in nature with common effects, e.g., observation of flocks of birds and swarms of insects motivated several studies in the past $[39,40]$. Humans display similar behavior in many instances, such as pedestrian motion, panic and vehicular traffic. Pedestrian behavior has been empirically studied since the 1950s [41]. The first physical modeling was proposed in [42] by a comparison with Navier-Stokes equations, but already in [25] it has been observed that in the dynamics of living entities energy and momentum are not generally conserved.

In more recent years, a new approach to behavioral-social dynamics for pedestrian crowds in the framework of the kinetic theory of active particles has been developed by dealing with the modeling of crowd dynamics to show that collective behaviors are induced by individual bases interactions $[43,44]$. The learning dynamics have an important role in crowd models as the walking strategy takes advantage of what each individual learns from the other walkers [45]. In this approach, the level of stress is not simply modeled as a parameter, but it is a variable whose dynamics is related to learning and social contagion. 


\subsection{Critical Analysis}

The mathematical modeling of collective learning should provide a characterization of all the fundamental aspects involved in the process and at the same time to be able to capture the fundamental aspects that determine the dynamical evolution of the system. The main feature to tackle is the complexity of the living systems. Indeed, the phenomenon we wish to describe can be represented considering a large collection of living entities which interact among them at microscopic level, through nonlinear interactions. The outcome of all the interactions determines the dynamical evolution of the overall system at a macroscopic level.

The aim of the present paper is to construct a mathematical framework suitable to capture the following aspects of the learning process:

- The individual's role in the collectivity. Collective learning modifies the individual's ability to develop interaction rules that evolve over time.

- Learning dynamics. Living entities can learn not only by micro-scale interactions, but also from the whole population. The key problem consists of referring the dynamics at a micro-scale to treat at the macro-scale.

- Role of networks. Networks can have an important influence on the learning dynamics, as observed in [9] both exogenous and endogenous networks have to be considered corresponding, respectively, to physically localized nodes and nodes generated by aggregations due to affinity principles.

\section{Mathematical Tools}

The derivation of mathematical models to describe the dynamics of collective learning is developed in two consecutive phases. Firstly, by defining a general structure capable of capturing the specific features of the class of systems under examination, and subsequently by creating models related to well defined learning phenomena. The derivation is obtained by refining the general structure by inserting into it models suitable to describe the interactions among the subjects involved in the learning dynamics. Examples of interactions between learning and social dynamics will be analyzed in the next section, while only methodological aspects are treated here.

Further conceptual developments are needed, with respect to [1], because our paper addresses complex dynamics for which learning is an important component, but only a preparatory step preceding other dynamics. In fact, in addition to the variables that characterize the learning process, it is also necessary to include more variables characterizing the induced dynamics.

This brief introduction defines the content of this section that will be dealt with in the following subsections addressed to the main three themes:

1. Characterization of the soft variables and representation of the system;

2. Modeling of interactions;

3. Derivation of a general structure suitable to describe the collective dynamics of interactions between learning and social dynamics.

The study will be limited to the case where the microscopic variable is defined by two components, i.e., learning and social. The methodological approach can then be generalized technically in the case of a larger number of components as discussed in the last section of our paper. Hereafter, we will use the following terminology: active particles (a-particles) to identify interacting entities; activity to indicate their status at the microscopic scale (individual based); and functional subsystems (FSs) with reference to the groups in which the population is divided.

\subsection{Soft Variables and Representation}

We consider a system of a large number of interacting entities with the following features:

- The system is composed by living entities which interact in a spatially homogeneous dynamics; 
- $\quad$ The microscopic scale corresponds to the state of each entity modeled as an a-particle [4];

- Each a-particle interacts with the others at both the microscopic level and the collective set of all the particles.

Therefore, according to [4], we introduce the activity, which is a vector variable with two components $\mathbf{w}=\{u, v\}$, where $u$ is the component that characterizes the level of learning and $v$ is the social component whose dynamics are also induced by $u$. Both $u$ and $v$ can be defined, according to an occasionally used jargon, soft variables.

In principles, although accounting for Sigmund's important book [46], soft variables are not measurable. However, we can conjecture and identify the minimum and maximum values that these variables may reach by defining a suitable interval where values are observed. Therefore, normalizing with respect to that domain it is obtained: $u \in[0,1] \equiv D_{u}$ and $v \in[0,1] \equiv D_{v}$. Moreover, local measures cannot be effectively made, therefore it is convenient using discrete, rather than continuous, variables.

For instance, a discrete, equally spaced collocation, can be used for the components $u$ and $v$ :

$$
C_{u}=\left\{u_{1}=0, \ldots, u_{n}=1\right\}, \quad \text { and } \quad C_{v}=\left\{v_{1}=0, \ldots, v_{n}=1\right\} .
$$

Therefore, the representation is delivered by the discrete probability distributions

$$
\mathbf{f}=\left\{f_{i j}(t)=f\left(u_{i}, v_{j} ; t\right)\right\} \quad \text { with } \sum_{i=1}^{n} \sum_{j=1}^{n} f_{i j}(t)=1 .
$$

If $f_{i j}(t)$ is known, p-order moments are computed by weighted sums:

$$
\mathbb{E}^{p}\{u\}(t)=\frac{1}{n} \sum_{i=1}^{n} \sum_{j=1}^{n} u_{i}^{p} f_{i j}(t) \text { and } \mathbb{E}^{p}\{v\}(t)=\frac{1}{n} \sum_{i=1}^{n} \sum_{j=1}^{n} v_{j}^{p} f_{i j}(t) .
$$

If active particles are subdivided into $m$ FSs, the following notation is used:

$$
\mathbf{f}^{r}=\left\{f_{i j}^{r}(t)=f^{r}\left(u_{i}, v_{j} ; t\right)\right\} \quad r=1, \ldots, m .
$$

Our paper refers to systems where the total number of particles $N$ is constant in time, so that the normalization condition is written as follows:

$$
\sum_{r=1}^{m} \sum_{i=1}^{n} \sum_{j=1}^{n} f_{i j}^{r}(t)=1
$$

where each $f_{i j}^{r}$ is a component of a probability as it has been divided by $N$.

\subsection{Modeling Interactions}

The first step of the modeling of interactions consists of selecting those interactions that play a non-trivial role in the dynamics of the system, while the second step consists of taking into account also a possible hierarchy among the activities $u$ and $v$, and eventually in modeling the output of interactions. The stochastic game theory [4] describes their dynamics by means of the frequency of the interactions and the transition probability of microscopic states of the interacting active particles which pursue a specific payoff consistent with the strategy they are able to develop. Moreover, rather than individual entities chasing their own pay-off, the whole population operates to achieve a collective well-being, where the advantage is shared by everyone. This concept is well expressed by the following quotation from Ref. [5]:

Evolutionary game theory deals with an entire population of players, all programmed to use the same strategy (or type of behavior). Strategies with higher payoff will spread within the population (this can 
be achieved by learning, by copying or inheriting strategies, or even by infection). The payoffs depend on the actions of the co-players and hence on the frequencies of the strategies within the population. Since these frequencies change according to the payoffs, this leads to a feedback loop. The dynamics of this feedback loop is the object of evolutionary game theory.

This subsection shows how interactions can be described by suitable operators, to be subsequently used in the derivation of the mathematical structure we are looking for. According to the kinetic theory of active particles, the dynamics of interactions involve candidate, field, and test active particles. In detail, we consider:

- Test particles of the $r$-FS with microscopic state $u_{i}, v_{j}$ and probability $f_{i j}^{r}(t)$. These particles are assumed to be representative of the whole system.

- Field particles of the $r$-FS with microscopic state $u_{p}, v_{q}$ and probability $f_{p q}^{r}(t)$ which are generic particles for each $r$-FS.

- Candidate particles, of the $k$-FS with microscopic state $u_{h}, v_{k}$ and probability $f_{h k}^{k}(t)$ which are generic particles for each $k$-FS deemed to take, due to interactions, the state of the test particles.

The modeling of learning interactions can be achieved by the interaction rate which describes the number of encounters per unit time, and the transition probability which denotes the probability that a candidate particle takes the state of the test particle due to interaction with field particles.

Dealing with these modeling issues leads straightforwardly to derive the governing evolution equation which regulates the dynamics of the class of systems under consideration. If we consider the simple case of one FS only, a formal expression of the aforementioned terms is as follows:

$\eta_{h k}^{p q}$ is the interaction rate of a $h k$-particle with a $p q$-particle, namely between particles with states $h k$ and $p q$.

$\mu_{h k}$ is the interaction rate of a $h k$-particle with the mean state $\mathbb{E}$ of the whole system.

$\mathcal{A}_{h k}^{p q}(h k \rightarrow i j)$ models the transition of a candidate $h k$-particle into the state of the test $i j$-particle due to the interaction with a field $p q$-particle.

$\mathcal{M}_{h k}(h k \rightarrow i j)$ models the transition of a candidate $h k$-particle into the state of the test $i j$-particle due to the interaction with the mean value within the system.

A balance of particles in the elementary volume of the microscopic state yields:

$$
\begin{aligned}
\frac{d}{d t} f_{i j} & =\sum_{h, k, p=1}^{n} \eta_{h k}^{p q}(\mathbf{f}) \mathcal{A}_{h k}^{p q}(h k \rightarrow i j)(\mathbf{f}) f_{h k} f_{p q}-f_{i j} \sum_{p, q=1}^{n} \eta_{i j}^{p q}(\mathbf{f}) f_{p q} \\
& +\sum_{h, k=1}^{n} \mu_{h k}(\mathbf{f}) \mathcal{M}_{h k}(h k \rightarrow i j)(\mathbf{f}) f_{h k} \mathbb{E}(\mathbf{f})-f_{i j} \mu_{i j}(\mathbf{f}) \mathbb{E}(\mathbf{f}),
\end{aligned}
$$

where all interaction terms might depend on $\mathbf{f}$, while the dependent variables are a function of time.

Analogous calculations can be developed if the system is constituted by a number $m$ of interacting functional subsystems:

$$
\begin{aligned}
\frac{d}{d t} f_{i j}^{r}= & \sum_{s=1}^{m} \sum_{h, k, p, q=1}^{n} \eta_{h k}^{p q}(r, s)(\mathbf{f}) \mathcal{A}_{h k}^{p q}(h k \rightarrow i j)(\mathbf{f}) f_{h k}^{r} f_{p q}^{s}-f_{i j}^{r} \sum_{s=1}^{m} \sum_{p, q=1}^{n} \eta_{i j}^{p q}(\mathbf{f}) f_{p q}^{s} \\
& +\sum_{s=1}^{m} \sum_{h, k,=1}^{n} \mu_{i j}^{s}(\mathbf{f}) \mathcal{M}_{h k}^{s}(h k \rightarrow i j)(\mathbf{f}) f_{h k} \mathbb{E}_{s}(\mathbf{f})-f_{i j}^{r} \sum_{s=1}^{m} \mu_{i j}(\mathbf{f}) \mathbb{E}_{s}(\mathbf{f}) .
\end{aligned}
$$

Still following the methodological style of presentation of our paper, some indications can be given to show how a mathematical description of interactions leads to the derivation of the models. Mainly qualitative indications are provided, leaving a detailed formalization to specific models.

Let us indicate how a possible interaction hierarchy can account for the sequential combination of the two different dynamics, namely learning, which corresponds to the variable $u$, and the subsequent 
dynamics corresponding to $v$. We propose, without claiming full generality, an approach grounded on the assumption that dynamics of the learning component $u$ precedes and is independent of the following dynamics, which, however, also depends on $u$. This assumption is not the most general one, but it can cover. The modeling of the interaction rates and of the transition probability can account, specifically, on the said assumption.

Let us now consider the modeling of the interaction rate which requires the definition of different concepts of distance between interacting entities. Some concepts can be given, which can be applied both to the distance between candidate and field particles and to that between test and field particles. The hierarchy indicates that interactions within the same FS can be considered in sequence. Therefore, the aforementioned distance can be considered: The micro-micro distance refers to the individual microscopic states within the same functional subsystems, namely $\left|u_{i}-u_{p}\right|$ and $\left|v_{j}-v_{q}\right|$, where the subscripts $p$ and $q$ refer to the field particles in the same functional subsystem; and the micro-macro distance refers to the interaction between the individual micro-state and the whole FS whose state is defined through a suitable average, for instance the mean value. Then, one has that micro-macro distances are given by $\left|u_{i}-\mathbb{E}^{s}\right|$ and $\left|v_{j}-\mathbb{E}^{s}\right|$. As a consequence, the interaction rate can be modeled by assuming that the interaction rate decays with the distance, between the interacting entities, measured by a specific metrics. In more detail, the metrics can be obtained by an exponential decay with a distances, either micro-micro or micro-macro from a basic value delivered when the distance is equal to zero. Symmetric interactions appear if interaction rates depend on the metrics defined above, while asymmetric interactions can be modeled by introducing a weight function depending on the localization of the nodes.

Consider now the modeling of the transition probability which models the output of the interaction of a-particles, with state $u_{h}, v_{k}$ within the i-th FS due to interaction with other a-particles as well as with the FS as a whole. The aforementioned hierarchy implies that the dynamics of the learning component $u$ precedes and is independent of the dynamics of the social component $v$, whose interaction dynamics, however, depend on $u$. For this reason, the transition probability $\mathcal{A}$ is composed of the contribution of two transition probabilities, namely the first one modeling the change of the level of knowledge of the candidate particle and a second one which accounts for the subsequent dynamics conditioned by the learning level. Therefore, the transition probability can be given as a factorization of the transition probabilities over the discrete variables $u$ and $v$.

The role of exogenous networks can be taken into account by the selection of the functional subsystem to be related to the localization of the nodes as proposed in [47,48]; here, we use discrete probability rather than continuous probability density. The problem of the creation of endogenous networks generated by aggregations, which is one of the key problems posed in [49] cannot be straightforwardly treated by the approach of this paper as it needs additional work.

The specific applications treated in the next section aims at showing how the aforementioned general approach can be particularized towards specific case studies. In addition, it will be shown how the further developments will be motivated by well defined applications. It is worth mentioning that a common feature of all applications is the interaction of learning dynamics with a different type of dynamics which precedes or follows learning. In mode details, three case studies follow-first, in social dynamics, where welfare policy is an important influence over the learning dynamics underlying the opinion formation. Second, collective learning modifies the mechanical rules by which crowds move. Lastly, collective learning in multicellular systems modifies the proliferative and/or destructive interactions in the immune competition.

\section{Case Studies towards Perspectives}

The literature critically analyzed in Section 2 has enlightened a wide class of possible learning dynamics in view of a modeling approach. We do not naively claim that the methodological approach proposed in our paper can cover the aforementioned whole variety. Therefore, we will focus on the main objective of our description, namely the modeling of the interactions between learning dynamics 
and other types of dynamics which can be modeled by kinetic theory methods. This statement can be made more precise by selecting three case studies, which present different features, to show how the approach can be technically developed.

Firstly, we show how the learning dynamics, related to welfare distribution, can lead to a distribution of support/opposition to governments, which, in turn, can even generate radical opposition. Secondly, we refer to the interaction between learning and crowd dynamics, namely between learning which leads to mechanical dynamics, namely walking strategies. Lastly, we consider the immune competition in heterogenous multicellular systems, where learning leads to proliferative and destructive events. Well defined hints will be given for each of them in view of research programs which might take advantage of the suggestions of this section. As we shall see, some applications might require further developments of the approach proposed in Section 3 as we will critically analyze in the last subsection.

\subsection{Social Conflicts and Radical Opposition}

Let us firstly consider the modeling of a dynamics where a welfare policy can lead to social conflicts and radical opposition. In more detail, let us consider the dynamics treated in [8], where a certain welfare policy generates either support or opposition to the Government author of the said policy. We consider an opinion formation process driven by the learning dynamics by which individuals communicate and "learn". Already, the authors in [8] have shown that the modeling approach should account for nonlinearly additive interactions. This feature has been confirmed in some subsequent papers that have developed this pioneer idea, for instance [50-52]. The approach of [8] models the sequential interaction of two dynamics: wealth distribution which has been treated by various authors e.g., $[36,53,54]$ and opinion formation has been introduced in [37] and subsequently treated by various authors, for instance [55,56]. A survey of the literature on this topic has been reviewed in [57].

The modeling of wealth distribution precedes the modeling of support or opposition to a government. Our hint is that the modeling approach developed in [8] deserves a revisiting focused on a deep analysis on the role of learning dynamics. In particular, the following research objectives are proposed:

1. Modeling the role of the individual learning from the whole population to understand how a certain trend, which can be conditioned by the wealth distribution, can lead to radicalization of the political contrast.

2. Modeling the transition, which can be viewed as a Darwinist mutation from a radical, however democratic, opposition, to a segregation of individuals ready to break laws and bring their opposition up to extreme levels.

3. Modeling the selection following mutations by an approach which might require the addition of a new FS corresponding to security forces/actions to act against the aforementioned radicalization.

The learning dynamics presented in [8] were based on simple rules, while the modeling of the mutations towards radicalization can be developed by introducing a threshold in the radicalization level which identifies the transition to extremism.

\subsection{Collective Learning and Dynamics of Crowds}

The second case study refers to the interaction between learning dynamics which produces social behaviors and the dynamics of crowds which is a mechanical system, where human behaviors [44] appear. Learning can have an important influence over the walking strategy of individuals in the crowd as it is shown in a recent paper [45], where a modeling approach includes the propagation of stress conditions. In particular, it is shown how stress conditions can modify both the overall dynamics and density patterns, inducing the formation of overcrowded zones, which is a bad event against safety. The modeling approach proposed in [45] is based on tools of the kinetic theory initiated in [43] 
and further developed in [44], while the derivation of macroscopic equations from the underlying description at the microscopic scale delivered by kinetic models has been obtained [58,59].

This topic deserves further research developments which might take advantage of the following hints:

1. The development of a learning dynamics in a crowd, where individuals communicate and learn through small group awareness. Communications propagate stress, which in turn can generate unsafe dynamics.

2. An additional interesting example is that of swarm's dynamics where communications and learning induce a collective motion with flocking properties [60]. Such specific interaction between learning and mechanics might generate a motion suitable to optimize the swarm defense against the attack of predators [61].

3. Collective learning should include nonlocal effects that have not yet been treated in [1] nor, in full generality, in [45]. Therefore, this research perspective requires further developments of the mathematical theory proposed in Section 3 to account for space dynamics and pattern formation.

\subsection{Multicellular Systems and Immune Competition}

Lastly, let us consider the dynamics of the immune system versus cells carriers of a pathology. We consider, referring to [62], a multicellular system including three populations, namely epithelial, immune, and cancer cells. Each population has a different internal variable. Immune cells can proliferate and activate their internal variable which can correspond to the defence ability against cancer cells. Cancer cells can proliferate and progress towards states with higher pathological states, namely by increasing their proliferative ability up to metastatic competence [63,64]. Immune cells have the ability of depleting cancer cells, which, in opposition, can inhibit the defence activity of immune cells. The first learning process of the immune cells is the acquiring of immune competence that enriches the innate immunity [65].

These complex dynamics have been studied in $[62,66,67]$ which have been inspired by researchers in the field of immunology [68] who motivated their conjecture by the idea that learning dynamics can induce mutations both for cancer and immune cells. Subsequently, the dynamics of competition, which ends up with proliferative and destructive events, induces selection. In particular, biologists argue that the learning dynamics occurs not only across the competing species, but also within the same population. For instance, active immune cells can learn from inhibited immune cells, and similarly weakly progressing cancer cells can learn from highly progressing cancer cells.

The following hints are brought to the attention of the interested reader:

1. A possible research program would be a deep analysis of the learning dynamics and the related influence over the immune competition.

2. The approach with an additional difficulty consists of the modeling of a system with a variable number of interacting living entities as proliferative and/or destructive encounters that appear in this dynamics.

3. The number of interacting functional subsystem grows in time due to the onset, by mutations, of new subsystems.

The mathematical tools proposed in Section 3 appear to be consistent with the requirements of the first hint, while the second and third hint need developments of new theoretical tools.

\subsection{Critical Analysis towards Perspectives}

Our paper has shown how the kinetic theory approach to collective learning can be developed to model cross interactions between learning and subsequent dynamics. Three specific applications have been outlined focusing on different living systems, while possible hints have been given to develop these applications. Therefore, looking at research perspectives, it is quite natural to 
tackle the challenging problems presented in this section by taking advantage of the said hints. These applications indicate that the mathematical structures derived in Section 3 might need to be further developed to account for some aspects of the Darwinist dynamics, space propagation and proliferative/destructive events.

In addition to the above reasoning, it is worth mentioning that the approach cannot be limited to modeling, but it should also require development of computational tools. Considering that the approach refers to equations of the kinetic theory, Monte Carlo particle methods appear to be the approach consistent with the structures of these types of equations. Therefore, one has to deal with the development of pioneering works, for instance referring to the books $[69,70]$ to recent applications reported in $[36,71,72]$. Numerical simulations can also contribute to cover, however at a heuristic level, issues that are not easily tractable, such as existence of equilibrium configuration and related stability analysis.

Lastly, let us focus on the problem posed by a multiscale vision, where macro-scale models are derived from micro-scale models as delivered by kinetic type models. A unified approach to this specific multiscale problem has been proposed in $[73,74]$. It is a general analytic tool that can be further specialized to a micro-macro derivation for the class of systems treated in our paper.

Author Contributions: Conceptualization, D.B. and S.D.L.; Methodology, D.B. and S.D.L.; Writing-original draft, D.B.

Funding: This research received no external funding.

Conflicts of Interest: The authors declare no conflict of interest.

\section{References}

1. Burini, D.; De Lillo, S.; Gibelli, L. Collective learning modeling based on the kinetic theory of active particles. Phys. Life Rev. 2016, 16, 123-139. [CrossRef] [PubMed]

2. Burini, D.; De Lillo, S.; Gibelli, L. Learning dynamics towards modeling living systems: reply to comments on "Collective learning dynamics modeling based on the kinetic theory of active particles". Phys. Life Rev. 2016, 16, 158-162. [CrossRef] [PubMed]

3. Burini, D.; Gibelli, L.; Outada, N. A kinetic theory approach to the modeling of complex living systems. In Active Particles; Bellomo, N., Degond, P., Tadmor, E., Eds.; Birkhäuser: Basel, Switzerland, 2017; Volume 1, pp. 229-258.

4. Bellomo, N.; Bellouquid, A.; Gibelli, L.; Outada, N. A Quest towards a Mathematical Theory of Living Systems; Birkhäuser: Basel, Switzerland, 2017.

5. Hofbauer, J.; Sigmund, K. Evolutionary game dynamics. Bull. Am. Math. Soc. 2003, 40, 479-519. [CrossRef]

6. Nowak, M.A. Evolutionary Dynamics. Exploring the Equations of Life; Harvard University Press: Cambridge, MA, USA, 2006.

7. Bellomo, N.; Knopoff, D.; Soler, J. On the difficult interplay between life, "complexity", and mathematical sciences. Math. Mod. Meth. Appl. Sci. 2013, 23, 1861-1913. [CrossRef]

8. Bellomo, N.; Herrero, M.A.; Tosin, A. On the dynamics of social conflicts: Looking for the Black Swan. Kinet. Relat. Models 2013, 6, 459-479.

9. Dolfin, M.; Leonida, L.; Outada, N. Modelling human behaviour in economics and social science. Phys. Life Rev. 2017, 22, 1-21. [CrossRef] [PubMed]

10. Vygotsky, L. Interaction between learning and development. Read. Dev. Child. 1978, 23, 34-41.

11. Piaget, J. "Piaget's Theory" Piaget and His School; Springer: Berlin/Heidelberg, Germany, 1976; pp. 11-23.

12. Bandura, A. Human agency in social cognitive theory. Am. Psychol. 1989, 44, 1175-1184. [CrossRef]

13. Cole, M.; Engestrom, Y. A cultural-historical approach to distributed cognition. In Distributed Cognitions: Psychological and Educational Considerations; Salomon, G., Ed.; Cambridge University Press: New York, NY, USA, 1993; pp. 1-46.

14. Munsterberg, H. Psychology, General and Applied; D. Appleton: Boston, MA, USA, 1915.

15. Gardner, H. The Mind's New Science: A History of the Cognitive Revolution; Basic Books: New York, NY, USA, 1987. 
16. Salomon, G.; Perkins, D.N. Individual and social aspects of learning. Rev. Res. Educ. 1998, 23, 1-24. [CrossRef]

17. Armitage, D.; Marschke, M.; Plummer, R. Adaptive co-management and the paradox of learning. Glob. Environ. Chang. 2008, 18, 86-98. [CrossRef]

18. Reed, M.; Evely, A.C.; Cundill, G.; Fazey, I.; Glass, J.; Laing, A.; Newig, J.; Parrish, B.; Prell, C.; Raymond, C.; et al. What is social learning? Ecol. Soc. 2010, 15, r1. [CrossRef]

19. Borowski, I.; Le Bourhis, J.; Pahl-Wostl, C.; Barraqué, B. Spatial misfit in participatory river basin management: Effects on social learning, a comparative analysis of German and French case studies. Ecol. Soc. 2008, 13, 7. [CrossRef]

20. Kuper, M.; Dionnet, M.; Hammani, A.; Bekkar, Y.; Garin, P.; Bluemling, B. Supporting the shift from state water to community water: Lessons from a social learning approach to designing joint irrigation projects in Morocco. Ecol. Soc. 2009, 14, 19. [CrossRef]

21. Pahl-Wostl, C.; Sendzimir, J.; Jeffrey, P.; Aerts, J.; Berkamp, G.; Cross, K. Managing change toward adaptive water management through social learning. Ecol. Soc. 2007, 12, 30. [CrossRef]

22. Davidson-Hunt, I.; Berkes, F. Learning as you journey: Anishinaabe perception of social-ecological environments and adaptive learning. Conserv. Ecol. 2003, 8, 5. [CrossRef]

23. Fazey, J.A.; Martonl, F. Understanding the space of experiential variation. Act. Learn. High. Educ. 2002, 3, 234-250. [CrossRef]

24. Esteva, A.; Kuprel, B.; Novoa, R.A.; Ko, J.; Swetter, S.M.; Blau, H.M.; Thrun, S. Dermatologist-level classification of skin cancer with deep neural networks. Nature 2017, 542, 115-118. [CrossRef]

25. Garavan, T.N.; Carbery, R. Collective Learning; Encyclopedia of the Sciences of Learning; Springer: Boston, MA, USA, 2012; pp. 646-649.

26. Capello, R. Spatial transfer of knowledge in high technology milieux: Learning versus collective learning processes. Reg. Stud. 1999, 33, 353-365. [CrossRef]

27. Dosi, G. Technological paradigms and technological trajectories: A suggested interpretation of the determinants and directions of technical change. Res. Policy 1982, 11, 147-162. [CrossRef]

28. Nelson, R.R.; Winter, S.G. An Evolutionary Theory of Economic Change; Harvard University Press: Cambridge, MA, USA, 1982.

29. Castellano, C.; Fortunato, S.; Loreto, V. Statistical physics of social dynamics. Rev. Mod. Phys. 2009, 81, 591. [CrossRef]

30. Weidlich, W. The statistical description of polarization phenomena in society. Br. J. Math. Stat. Psychol. 1971, 24, 251-266. [CrossRef]

31. Galam, S.; Gefen, Y.; Shapir, Y. Sociophysics: A new approach of sociological collective behaviour. I. mean-behaviour description of a strike. J. Math. Sociol. 1982, 9, 1-13. [CrossRef]

32. Galam, S.; Moscovici, S. Towards a theory of collective phenomena: Consensus and attitude changes in groups. Eur. J. Soc. Psychol. 1991, 21, 49-74. [CrossRef]

33. Holley, R.A.; Liggett, T.M. Ergodic theorems for weakly interacting infinite systems and the voter model. Ann. Probab. 1975, 3, 643-663. [CrossRef]

34. Clifford, P.; Sudbury, A. A model for spatial conflict. Biometrika 1973, 60, 581-588. [CrossRef]

35. Carrillo, J.A.; Fornasier, M.; Rosado, J.; Toscani, G. Asymptotic flocking dynamics for the kinetic Cucker-Smale model. SIAM J. Math. Anal. 2010, 42, 218-236. [CrossRef]

36. Pareschi, L.; Toscani, G. Interacting Multiagent Systems: Kinetic Equations and Monte Carlo Methods; Oxford University Press: New York, NY, USA, 2013.

37. Toscani, G. Kinetic models of opinion formation. Commun. Math. Sci. 2006, 4, 481-496. [CrossRef]

38. Axelrod, R. The dissemination of culture: A model with local convergence and global polarization. J. Confl. Resolut. 1997, 41, 203-226. [CrossRef]

39. Czirók, A.; Barabási, A.L.; Vicsek, T. Collective motion of self-propelled particles: Kinetic phase transition in one dimension. Phys. Rev. Lett. 1999, 82, 209-212. [CrossRef]

40. Parrish, J.K.; Hamner, W.M. Animal Groups in Three Dimensions: How Species Aggregate; Cambridge University Press: Cambridge, UK, 1997.

41. Hankin, B.D.; Wright, R.A. Passenger flow in subways. J. Oper. Res. Soc. 1958, 9, 81-88. [CrossRef]

42. Henderson, L.F. The statistics of crowd fluids. Nature 1971, 229, 381-383. [CrossRef] [PubMed] 
43. Bellomo, N.; Bellouquid, A.; Knopoff, D. From the microscale to collective crowd dynamics. Multiscale Model. Simul. 2013, 11, 943-963. [CrossRef]

44. Bellomo, N.; Gibelli, L. Toward a mathematical theory of behavioral-social dynamics for pedestrian crowds. Math. Mod. Meth. Appl. Sci. 2015, 25, 2417-2437. [CrossRef]

45. Bellomo, N.; Gibelli, L.; Outada, N. On the interplay between behavioral dynamics and social interactions in human crowds. Kinet. Relat. Models 2019, 12, 397-409. [CrossRef]

46. Sigmund, K. The Calculus of Selfishness; Princeton University Series in Theoretical and Computational Biology; Princeton University Press: Princeton, NJ, USA, 2011.

47. Knopoff, D. On the modeling of migration phenomena on small networks. Math. Mod. Meth. Appl. Sci. 2013, 23, 541-563. [CrossRef]

48. Knopoff, D. On a mathematical theory of complex systems on networks with application to opinion formation. Math. Mod. Meth. Appl. Sci. 2014, 24, 405-426. [CrossRef]

49. Bonacich, P.; Lu, P. Introduction to Mathematical Sociology; Princeton University Press: Princeton, NJ, USA, 2012.

50. Dolfin, M.; Lachowicz, M. Modeling altruism and selfishness in welfare dynamics: The role of nonlinear interactions. Math. Mod. Meth. Appl. Sci. 2014, 24, 2361-2381. [CrossRef]

51. Dolfin, M.; Lachowicz, M. Modeling opinion dynamics: How the network enhances consensus. Netw. Heterog. Media 2015, 10, 421-441. [CrossRef]

52. Dolfin, M.; Knopoff, D.; Leonida, L.; Maimone Ansaldo Patti, D. Escaping the trap of 'blocking': A kinetic model linking economic development and political competition. Kinet. Relat. Models 2017, 10, 423-443. [CrossRef]

53. Bertotti, M.L.; Delitala, M. From discrete kinetic and stochastic game theory to modelling complex systems in applied sciences. Math. Mod. Meth. Appl. Sci. 2004, 14, 1061-1084. [CrossRef]

54. Bisi, M.; Spiga, G.; Toscani, G. Kinetic models of conservative economies with wealth redistribution. Commun. Math. Sci. 2009, 7, 901-916. [CrossRef]

55. Aletti, G.; Naldi, G.; Toscani, G. First-order continuous models of opinion formation. SIAM J. Appl. Math. 2007, 67, 837-853. [CrossRef]

56. Caponigro, M.; Lai, A.C.; Piccoli, B. A nonlinear model of opinion formation on the sphere. Discret. Cont. Dyn. Syst. Ser. A 2015, 35, 4241-4268.

57. Aimone Marsan, G.; Bellomo, N.; Gibelli, L. Stochastic evolutionary differential games toward a systems theory of behavioral social dynamics. Math. Mod. Meth. Appl. Sci. 2016, 26, 1051-1093. [CrossRef]

58. Bellomo, N.; Bellouquid, A. On multiscale models of pedestrian crowds from mesoscopic to macroscopic. Commun. Math. Sci. 2015, 13, 1649-1664. [CrossRef]

59. Bellomo, N.; Bellouquid, A.; Nieto, J.; Soler, J. On the multiscale modeling of vehicular traffic: From kinetic to hydrodynamics. Discret. Cont. Dyn. Syst. B 2014, 19, 1869-1888. [CrossRef]

60. Bellomo, N.; Ha, S.Y. A quest toward a mathematical theory of the dynamics of swarms. Math. Mod. Meth. Appl. Sci. 2017, 27, 745-770. [CrossRef]

61. Di Francesco, M.; Fagioli, S. A nonlocal swarm model for predators-prey interactions. Math. Mod. Meth. Appl. Sci. 2016, 26, 319-355. [CrossRef]

62. Bellouquid, A.; De Angelis, E.; Knopoff, D. From the modeling of the immune hallmarks of cancer to a black swan in biology. Math. Mod. Meth. Appl. Sci. 2013, 22, 949-978. [CrossRef]

63. Hanahan, D.; Weinberg, R.A. Hallmarks of cancer: The next generation. Cell 2011, 144, 646-674. [CrossRef]

64. Weinberg, R.A. The Biology of Cancer; Garland Sciences-Taylor and Francis: New York, NY, USA, 2007.

65. Cooper, E.L. Evolution of immune system from self/not self to danger to artificial immune system. Phys. Life Rev. 2010, 7, 55-78. [CrossRef] [PubMed]

66. Bellouquid, A.; Delitala, M. Mathematical Modeling of Complex Biological Systems; Birkhäuser: Basel, Switzerland, 2006.

67. De Angelis, E. On the mathematical theory of post-Darwinian mutations, selection, and evolution. Math. Mod. Meth. Appl. Sci. 2014, 24, 2723-2742. [CrossRef]

68. Cavallo, F.; De Giovanni, C.; Nanni, P.; Forni, G.; Lollini, P.L. 2011: The immune hallmarks of cancer. Cancer Immunol. 2011, 60, 319-326. [CrossRef] [PubMed]

69. Aristov, V.V. Direct Methods for Solving the Boltzmann Equation and Study of Nonequilibrium Flows; Springer: New York, NY, USA, 2001. 
70. Bird, G.A. Molecular Gas Dynamics and the Direct Simulation of Gas Flows; Oxford University Press: Oxford, UK, 1994.

71. Albi, G.; Bellomo, N.; Fermo, L.; Ha, S.-Y.; Kim, J.; Pareschi, L.; Poyato, D.; Soler, J. Traffic, crowds, and swarms. From kinetic theory and multiscale methods to applications and research perspectives. Math. Mod. Meth. Appl. Sci. 2019, 29. [CrossRef]

72. Barbante, P.; Frezzotti, A.; Gibelli, L. A kinetic theory description of liquid menisci at the microscale. Kinet. Relat. Mod. 2015, 8, 235-254.

73. Burini, D.; Chouhad, N. Hilbert method toward a multiscale analysis from kinetic to macroscopic models for active particles. Math. Mod. Meth. Appl. Sci. 2017, 27, 1327-1353. [CrossRef]

74. Burini, D.; Chouhad, N. A multiscale view of nonlinear diffusion in biology: From cells to tissues. Math. Mod. Meth. Appl. Sci. 2019, 29, 791-823. [CrossRef]

(C) 2019 by the authors. Licensee MDPI, Basel, Switzerland. This article is an open access article distributed under the terms and conditions of the Creative Commons Attribution (CC BY) license (http://creativecommons.org/licenses/by/4.0/). 